\title{
Development and Evaluation of an Accelerometer-Based Protocol for Measuring Physical Activity Levels in Cancer Survivors: Development and Usability Study
}

Tracy E Crane ${ }^{1^{*}}, \mathrm{PhD}, \mathrm{RDN}$; Meghan B Skiba ${ }^{2 *}, \mathrm{MS}, \mathrm{RDN}$; Austin Miller ${ }^{3}, \mathrm{PhD}$; David O Garcia ${ }^{2}, \mathrm{PhD}, \mathrm{FACSM}$; Cynthia A Thomson ${ }^{2}$, PhD, RDN

\footnotetext{
${ }^{1}$ Department of Biobehavioral Health Sciences, College of Nursing, University of Arizona, Tucson, AZ, United States

${ }^{2}$ Department of Health Promotion Sciences, Mel and Enid Zuckerman College of Public Health, University of Arizona, Tucson, AZ, United States

${ }^{3}$ Department of Biostatistics, Roswell Park Cancer Institute, Buffalo, NY, United States

*these authors contributed equally
}

\section{Corresponding Author:}

Tracy E Crane, PhD, RDN

Department of Biobehavioral Health Sciences

College of Nursing

University of Arizona

1305 N Martin Ave

Tucson, AZ, 85721

United States

Phone: 15203310120

Email: tecrane@email.arizona.edu

\section{Abstract}

Background: The collection of self-reported physical activity using validated questionnaires has known bias and measurement error.

Objective: Accelerometry, an objective measure of daily activity, increases the rigor and accuracy of physical activity measurements. Here, we describe the methodology and related protocols for accelerometry data collection and quality assurance using the Actigraph GT9X accelerometer data collection in a convenience sample of ovarian cancer survivors enrolled in GOG/NRG 0225, a 24-month randomized controlled trial of diet and physical activity intervention versus attention control.

Methods: From July 2015 to December 2019, accelerometers were mailed on 1337 separate occasions to 580 study participants to wear at 4 time points (baseline, 6, 12, and 24 months) for 7 consecutive days. Study staff contacted participants via telephone to confirm their availability to wear the accelerometers and reviewed instructions and procedures regarding the return of the accelerometers and assisted with any technology concerns.

Results: We evaluated factors associated with wear compliance, including activity tracking, use of a mobile app, and demographic characteristics with chi-square tests and logistic regression. Compliant data, defined as $\geq 4$ consecutive days with $\geq 10$ hours daily wear time, exceeded $90 \%$ at all study time points. Activity tracking, but no other characteristics, was significantly associated with compliant data at all time points $(P<.001)$. This implementation of data collection through accelerometry provided highly compliant and usable activity data in women who recently completed treatment for ovarian cancer.

Conclusions: The high compliance and data quality associated with this protocol suggest that it could be disseminated to support researchers who seek to collect robust objective activity data in cancer survivors residing in a wide geographic area.

(JMIR Mhealth Uhealth 2020;8(9):e18491) doi: 10.2196/18491

\section{KEYWORDS}

wearable electronic devices; physical activity; cancer survivors; activity trackers; mobile phone 


\section{Introduction}

\section{Background}

For cancer survivors, it has been demonstrated that physical activity has positive effects on psychosocial and physical outcomes, including weight management, quality of life, fatigue, emotional well-being, and sleep as well as social, cognitive, and physical functioning [1-3]. However, self-reported levels of physical activity among ovarian cancer survivors are low, with approximately $20 \%$ of women meeting the recommended 150 min of moderate-to-vigorous physical activity per week [4,5]. Measurement and assessment of physical activity in cancer survivors remain a challenge in randomized controlled trials, wherein self-reported questionnaires are most commonly used [6]. Subjective self-report of physical activity is wrought with significant recall and measurement error bias, as many study participants overreport moderate- and vigorous-intensity physical activities [7]. In previous trials, participants not only overreported physical activity but also there was a concurrent tendency to underreport sedentary time [8]. Furthermore, many self-report instruments fall short in capturing light-intensity physical activity [9], a major source of activity in adults, particularly cancer survivors [10]. In fact, in the Women's Health Study, fewer than $50 \%$ of women met physical activity guidelines as measured using accelerometry compared with approximately $67 \%$ women from self-reported physical activity [11]. Objective measurement of activity is, therefore, considered the gold standard for assessing physical activity exposure for all levels of intensity (rest, sedentary, light intensity, and moderate-to-vigorous intensity) in clinical trials and epidemiological studies.

Accelerometry provides the opportunity to objectively evaluate the minutes of activity per day, intensity, and total energy expenditure of physical activity as well as sedentary time, known factors associated with cancer risk [12,13]. Accelerometer protocols have been successfully implemented in population-level surveillance studies [10]. However, the existing information related to distance accelerometer methods (eg, mail based) has suggested poor compliance, with minimal improvement in recent years despite the growing use of this technique in assessing physical activity [14]. The use of accelerometry to capture objective activity data is particularly scarce among randomized and specifically lifestyle intervention trials in cancer survivors. A recent review highlighted that methods of accelerometry data collection among cancer survivors varied and were inconsistent, with limited studies reporting necessary details regarding data collection, compliance, and processing of data that would support replication of the research [15]. Robust measurement of physical activity using objective methods in cancer survivors is of high importance. Cancer survivors have unique needs as a result of cancer and subsequent treatment, including but not limited to fatigue, ostomies, abdominal pain, and chemotherapy-induced peripheral neuropathy $[4,16,17]$, all of which can be a barrier to physical activity.

\section{Objectives}

To improve the rigor of physical activity assessment in both epidemiologic and intervention studies, a standardized protocol for collecting reliable and valid mail-based accelerometer data is warranted. In this paper, we describe a mail-based protocol for prospective collection of accelerometry data in a convenience sample of 580 ovarian cancer survivors enrolled in a diet and physical activity intervention. Of note, study participants resided in 48 US states, suggesting that this protocol may be applicable and implemented in studies that recruit participants across wider geographic areas. Finally, the protocol was tested across multiple study time points to demonstrate compliance over time, a critical aspect of longitudinal research.

\section{Methods}

\section{Wearable Electronic Device}

The Actigraph GT9X Link is a validated triaxial accelerometer [18] that includes a gyroscope, magnetometer, secondary accelerometer, and Bluetooth capability [19] manufactured by ActiGraph, LLC. The Actigraph GT9X Link uses the same validated algorithms from its predecessor, GT3X, which was validated through indirect calorimetry [20]. Compared with GT3X, GT9X Link captures different step counts [21] but still provides comparable data and estimates of activity intensity and sedentary time [22]. Few studies have implemented GT9X Link to assess physical activity to date, but none have been in cancer survivors [23-25].

Physical activity was measured within the implementation of the GOG/NRG 0225 Lifestyle Intervention for oVarian cancer Enhanced Survival (LIvES) study (NCT00719303). Briefly, the LIvES study is a randomized controlled trial that tested a 24-month lifestyle intervention (high vegetable and fiber, low-fat diet with daily physical activity goals) compared with an attention control (general health education) on ovarian cancer progression-free survival. Eligible participants were in 6 weeks to 6 months postcancer treatment for stages II to IV disease and were randomized in a ratio of 1:1 to intervention versus control groups, stratified by receipt of consolidation therapy. Women were enrolled at NRG Oncology clinic sites nationwide. Intervention components are delivered via telephone by trained health coaches from the University of Arizona Cancer Center (UACC). Protocol and related methodologies as well as retention approaches for this study were previously published [26]. Measures included self-report physical activity assessment via the validated Arizona Physical Activity Questionnaire (APAQ) [27] and repeat accelerometer-measured activity at time points aligned with the self-report measure (baseline, 6 months, 12 months, and 24 months) as a measure of intervention adherence. The APAQ also included self-report of participants' height and weight, which was used to calculate their BMI. Participant demographics were collected using a standardized form at enrollment.

\section{Accelerometer Protocol}

The accelerometer protocol was developed using manufacturer instructions, a review of published literature [28-32], and prior experience of study investigators. The protocol development 
process included the creation of standardized instructional materials, a staff training program, implementation strategies, and data quality assessments. The objective activity was assessed using the GT9X Link accelerometer at 4 study time points: baseline, 6 months, 12 months, and 24 months (Figure 1).
Participants were asked to wear GT9X Link on their nondominant hip for a continuous period of 7 days, 24 hours a day, with the exception of water-based activities such as showering or swimming.

Figure 1. Participant communication schema for accelerometry data capture in the Lifestyle Intervention for oVarian cancer Enhanced Survival study.

\begin{tabular}{|c|c|c|}
\hline Staff & Participant & Staff \\
\hline $\begin{array}{c}\text { Coordinator calls to } \\
\text { determine participant } \\
\text { availability to wear } \\
\downarrow \\
\text { Initialize GT9X Link } \\
\downarrow \\
\text { Assemble package } \\
\downarrow \\
\text { Mails package via 2- } \\
\text { day tracked shipping }\end{array}$ & $\begin{array}{c}\text { Wears GT9X Link } \\
\text { for } 7 \text { consecutive } \\
\text { days, } 24 \text { hours a day, } \\
\text { removes before } \\
\text { engaging in water- } \\
\text { based activity } \\
\downarrow \\
\text { Completes tracking } \\
\text { log or uploads data } \\
\downarrow \\
\text { Returns prepaid } \\
\text { package via 2-day } \\
\text { tracked shipping }\end{array}$ & $\begin{array}{c}\text { Downloads data from } \\
\text { GT9X Link } \\
\downarrow \\
\text { Processes data } \\
\downarrow \\
\text { Coordinator } \\
\text { completes quality } \\
\text { assurance and control } \\
\text { to determine } \\
\text { compliant wear data }\end{array}$ \\
\hline
\end{tabular}

\section{Participant Instructional Materials}

The instructional materials for this protocol included a cover letter that summarized the procedures, guidance on wear, troubleshooting, and tracking logs for wear time. Before implementing the protocol, study staff and 2 individuals outside of the study (who were of the same age as study participants) beta-tested the materials to determine usability and acceptability of the instructional packet, tracking log, and mobile device app. Feedback from usability and acceptability testing was incorporated into the final study documents. The instruction packet was revised, as technology related to GT9X Link (such as chargers and waist clips) was updated in the inventory by ActiGraph.

An introductory letter was included in each package that oriented participants to the activity monitor portion of the study and acknowledged and thanked them for their study participation to date, specifically in relation to data collection at prior time points. The letter detailed the list of materials included in the mailed package and provided a study telephone number to call if they had any questions. The instructional booklet served as a resource for participants and included written instructions with photos that described how to (1) charge the activity monitor, (2) wear the activity monitor on the nondominant hip, (3) complete the tracking log, (4) download the CentrePoint Study Admin Sync app on a mobile device for data upload, and (5) prepare the package for return to the data collection center at the end of the 7-day wear period. At baseline, for participants who provided an email address, an instructional video that detailed the same information contained in the written instructional booklet was sent via email with the embedded web link. A monthly calendar was provided as a resource for participants to mark which days of the week the activity monitor was required to be worn and the date to return the accelerometer. Finally, a checklist of related Actigraph GT9X Link wear procedures was included as a tool to remind the participants of each step in the process and to promote inclusion of the activity log in the return package to the UACC Behavioral Measurement and Interventions Shared Resource (BMISR).

\section{Self-Monitoring}

A tracking log was provided to all participants to self-monitor wear time and record all accelerometer wear time on a daily basis for the 7-day wear period. The tracking log detailed self-reported measures of wake and sleep times as well as removal times of the accelerometer and indicated if the data were uploaded via the CentrePoint Study Admin Sync app. These data were used as quality assurance measures to determine the correct wear times for individual participants (which were then verified by accelerometer readings) as well as compliance with self-monitoring behaviors. Engagement with the CentrePoint mobile app, defined as a mobile upload of data during the 7-day wear period at least once, was confirmed by the researcher through the backend of the CentrePoint mobile app.

\section{Staff Training}

Study staff working with accelerometer data collection were trained and adhered to a standard operating procedure (SOP), specifically created for objective physical activity data collection in the context of the study. The SOP included guidance for staff in relation to initializing the accelerometer and downloading data from the Actigraph GT9X Link as well as the procedure for contacting participants (eg, phone script and number of call attempts) and mailing the packages. These SOPs were informed 
by previous studies that used the Actigraph GT9X Link accelerometers for the collection of physical activity data and further adapted for the study population in an effort to enhance compliance beyond prior reports [28-32].

\section{Mailing Approaches}

After confirming the availability to wear the accelerometer, the study staff created packages to send to the participant's home address. The packages included (1) introduction letter, (2) tracking log, (3) instruction packet, (4) charging pack, (5) return checklist, (6) monthly study activity calendar, and (7) return postage paid preaddressed return envelope. The United States Postal Service (USPS) Priority Flat Rate padded envelopes were used for sending and receiving packages. At the time of this study, USPS Priority mail provided 2-day shipping to the Continental United States, which was chosen to reduce the likelihood of an accelerometer losing charge in transit and expedite the wearing process to ensure that data could be collected in an appropriate time frame. USPS tracking numbers were assigned to each outgoing and incoming package and documented. Study staff would access updates of the location of the package through a web-based tool maintained by the USPS. The expected battery life of GT9X Link is 10 days; however, to ensure that battery life lasted through the duration of the shipping and wear period, a charging pack was included with the accelerometer. The window to open the accelerometer package and initiate wear time was \pm 2 weeks (14 days) at each time point. An extended window of an additional 4 weeks was used in extenuating circumstances such as extended travel, illness, or reissue of the accelerometer because of damage or loss. At the end of the wear duration, participants mailed the activity monitor, charging pack, and tracking log in the prepaid and addressed return envelope provided in the original package.

\section{Communication}

At each study time point, participants were contacted by trained study staff from UACC-BMISR via telephone. During this call, the participant was queried as to when and whether they would be available to wear the accelerometer for 7 consecutive days. If they were agreeable to wearing the accelerometer through verbal consent and confirmation, a follow-up call occurred within 2 days of confirmed delivery of the package to determine receipt by the participant and provide a detailed explanation of wear and documentation procedures. Predetermined wear dates were estimated by the study staff, including setting a return date estimated and documented as the last day of anticipated wear. An additional 4 days were added for return shipping. Participants were instructed to wear the accelerometer continuously for 24 hours a day for a total duration of 7 days, including during sleep, with the exception of when bathing, showering, or swimming. Participants were instructed to first fully charge the activity monitor upon receipt before wearing (and if the battery life dropped to $<10 \%$ ) and to start the wear time upon awakening the next day and continue to wear through wake time after day 7. For the duration of wear time, participants were requested to complete a tracking log that included date, time awake, time asleep, times they took off the device, and the reason the device was removed. If 1 of the wear days was missed, they were asked to wear the device for an additional day and document on the tracking log reason for nonwear (eg, clinic appointment, illness, and forgot).

A few areas of protocol implementation required troubleshooting to enhance compliance. The first was to reach the participant for the initial accelerometer distribution. Following the SOP, the study staff attempted to contact a participant twice a week for 2 consecutive weeks, after which the study coordinator would contact the clinic to ensure that contact information was correct before continuing further attempts to contact the participant. Second, select participants who delayed in returning the accelerometer. If an accelerometer was not received within 7 days of the anticipated return date, the participant would be called by the study coordinator and, if required, a voicemail was left. The expected return date was then updated. If still it was not received within 7 days of this extended return date, the participant was contacted again by telephone, and an email reminder was sent. If still not returned by 3 weeks after the initial expected return date, the oncology clinic where the participant gave consent for trial participation was contacted to confirm whether the participant was still active in the study and request that the clinic discusses the return of the accelerometer with the study participant during their next scheduled clinic visit. In addition, the study staff would attempt to contact the participant to return the accelerometer once a week for the next 4 weeks. If the accelerometer was not successfully returned after 2 consecutive months of contact attempts, the participant was considered protocol noncompliant, and a USPS-certified letter from the study's principal investigator was mailed to the participant's residential address to request the return of materials and provided instructions on how to do so.

\section{Data Collection and Quality Assessment}

\section{Data Collection and Capture}

The study staff tracked contact attempts with participants at each time point using an encrypted shared spreadsheet. This spreadsheet contained no personal identifying information, only the study participant ID numbers. Name, contact number, email, and mailing address were stored separately on the Health Insurance Portability and Accountability Act (HIPAA)-secure study platform and database [33]. The enrollment date of the participant was added to this spreadsheet and used to calculate the open windows for subsequent time points. It also included the initialization date of the accelerometer, mailing date, and tracking numbers of the packages as well as return date, download date, and whether or not compliant data were collected. Any special notes about the participant during the specified time point were documented on the spreadsheet. Of note, if a participant refused to wear or was unable to wear an accelerometer at one time point, this was documented, and they were still queried at subsequent time points if they remained active in the study. Refusal to wear was defined as the participant declining to wear the accelerometer for any reason (eg, not having available time, inconvenience, conflicts with religious holidays, or discomfort with wearing the accelerometer). Unable to wear was defined as participants expressing willingness to wear an accelerometer but not having physical capacity to wear during open windows (eg, surgery, hospitalization, illness or injury, or natural disaster). Participants 
were removed from being contacted at future study time points if the participant had reached a study endpoint (eg, disease progression, other diseases, lost to follow-up, or withdraw of participation) or was previously protocol noncompliant (eg, did not return the accelerometer after 2 consecutive months of contact attempts).

Data collection included the use of the Actigraph GT9X Link Study Admin Sync, a web-based study management platform for accelerometers. This platform permits participant connection through CentrePoint Study Admin Sync, a mobile app available on iOS and Android devices that gives participants the option to upload their accelerometer data in real time to a secure HIPAA-compliant cloud-based study database [34]. At the time of developing this protocol, there was no literature available regarding participant uptake of the use of this mobile app. Therefore, the integration of this platform was an opportunity to explore the use of a mobile app as a data collection tool to inform future research. Participants were asked whether they used a mobile device (eg, smartphone or tablet) and whether they would be willing to download the mobile app that connects the mobile device to the accelerometer through Bluetooth connectivity and upload the data through user response to the associated study database in the Actigraph GT9X Link Study Admin Sync. During the follow-up call, if the participant opted to use the mobile app, instructions for downloading and installing the app on their mobile device were covered in detail. To connect Actigraph GT9X Link to the app, a unique 5-digit code was given to the participant via telephone. Participants entered this code on their device within a 2-min time frame before the code expired. This mobile app was optional for the participant to upload their data at the end of each waking period.

In an attempt to rigorously collect objective physical activity data, provisions were made to reissue an accelerometer if the accelerometer was lost and data could be captured within the predesignated extended wear time frame window of 4 weeks. The same protocol for initializing and mailing the replacement accelerometer was followed.

All accelerometers and accessories were sanitized by the study staff upon return to UACC-BMISR. A separate spreadsheet was used by the study staff to track all accelerometers in a study-specific inventory. If a participant reported a problem with their accelerometer or charger, this was documented by the study staff and investigated upon return of the accelerometer. If the problem could not be resolved, the accelerometer or charging dock would be removed from the mailing rotation and replaced.

The accelerometers were initialized, and the data were downloaded using the Actigraph GT9X Link CentrePoint Study Admin System. Weight and age were entered for each participant during initialization, and the time zone of the participant was documented by the study staff. Participants were blinded to the physical activity feedback from the device; the screen was programmed to only display battery life, date, and time.

\section{Data Quality Assessment}

As part of quality control, the study staff visually inspected all processed data for compliance. Compliance with the accelerometer wear time was defined by the investigator group before study initiation. These cut-off points have been previously validated in adults; no specific cut-off points currently exist for cancer survivors [35]. Specifically, wear compliance was set at $\geq 4$ days, with $\geq 10$ hours of daily wear time [36,37]. Initial data from the accelerometer were downloaded and exported to ActiLife (version 6.12) software. Accelerometer data were processed using 60-second epochs and Freedson [38] cut-off points.

\section{Statistical Analysis}

Descriptive data are reported as frequency, means, or medians. Potential predictors of compliant data, including demographic characteristics and self-monitoring behavior, at baseline were evaluated using logistic regression models. As the sample size decreased longitudinally from participants reaching a predetermined study endpoint, logistic regression models were not repeated for subsequent time points. Comparison of factors related to self-monitoring behaviors and compliant accelerometry data collected at each time point were conducted using the Pearson chi-square tests. All statistics were completed in Stata 16 (StataCorp LLC).

\section{Results}

\section{Overview}

From July 2015 to December 2019, 580 cancer survivors active in the study were contacted by the study staff at enrollment to initiate the accelerometer procedures, $12.9 \%$ (75/580) provided an email address, and the majority preferred to communicate via telephone. Over the 4 time points, a total of 1337 individual accelerometer mailings were completed. At baseline, $98.4 \%$ (571/580) women were available and willing to wear the accelerometer-95.2\% (533/560) study participants were compliant with the wear protocol and had usable data, and 5\% (27/560) had insufficient wear time. For the 3.5\% (20/580) participants without the accelerometer wear time at baseline, reasons for nonparticipation included participants who refused to wear the accelerometer $(n=3)$, unable to wear because of illness or natural disasters $(n=8)$, and off-study before completing baseline assessments $(n=9)$.

At subsequent time points, participants who demonstrated protocol noncompliance (eg, not returning the accelerometer at a previous time point) were not asked to collect follow-up accelerometer data. In addition, if a participant reached a study endpoint, no further measurements of activity were completed. At 6 months, 95.2\% (394/414) of individual participants were sent accelerometers, resulting in the acquisition of compliant data from $90.9 \%$ (358/394) of the active sample. At 12 months, 260 active participants were sent accelerometers for repeat activity measurement, with $95.4 \%$ (248/260) of women providing compliant data. At 24 months, 123 eligible participants were sent accelerometers, resulting in $96.7 \%$ $(119 / 123)$ of the active sample that provided compliant data. 
The details of the accelerometer data compliance by time points are outlined in Table 1.

Across all time points, 49 accelerometers required a reissue for participant data collection. The majority $(32 / 49,65 \%)$ of the reissued accelerometers were at baseline. Of these reissues, $25 \%$ (12/49) were because of depleted battery life upon arrival, 20\% (10/49) for software malfunction, and 20\% (10/49) were lost by either the participant or in transit. Removal from mailing rotation and replacement of inventory for accelerometers that encountered issues and nonfunctional charging docks were 16 and 67, respectively.

Participants engaged in the accelerometer protocol were representative of the overall Lifestyle Intervention for oVarian cancer Enhanced Survival study population in terms of demographic and clinical characteristics [26]. On average, participants were aged 60.1 (SD 9.3) years, and the majority were non-Hispanic college graduates with a normal BMI (Table 2). The results from logistic regression models of age, education, ethnicity, and BMI as well as self-monitoring behaviors indicated that tracking log completion was the greatest predictor of compliant data at baseline (Table 3). Odds ratios (ORs) were significantly higher (OR 54.71, 95\% CI 17.05-175.59; $P<.001$ ) for having compliant data if a participant completed a corresponding tracking log compared with those who did not complete the tracking log during the 7-day wear period. Other factors, including age, education, ethnicity, BMI, and mobile app engagement, were not significantly associated with compliant data at baseline.

Table 1. Details of the accelerometer wear time compliance in 580 participants by time points on the Lifestyle Intervention for oVarian cancer Enhanced Survival accelerometer protocol.

\begin{tabular}{|c|c|c|c|c|}
\hline Study sample ${ }^{\mathrm{a}}$ & Baseline $^{\mathrm{a}}, \mathrm{n}(\%) ; \mathrm{N}$ & 6 months $^{\mathrm{a}}, \mathrm{n}(\%) ; \mathrm{N}$ & 12 months ${ }^{\mathrm{a}}, \mathrm{n}(\%) ; \mathrm{N}$ & 24, months $^{\mathrm{a}}, \mathrm{n}(\%) ; \mathrm{N}$ \\
\hline Total active ${ }^{b}$ sample & 571 (98.4); 580 & 414 (71.4); 580 & 284 (49.0); 580 & 154 (28.7); 580 \\
\hline Agree to wear ${ }^{c}$ & 560 (98.1); 571 & 394 (95.2); 414 & $260(91.5) ; 284$ & 123 (79.9); 154 \\
\hline Compliant data $^{\mathrm{d}}$ & $533(95.2) ; 560$ & 358 (90.9); 394 & $248(95.4) ; 260$ & $119(96.7) ; 123$ \\
\hline Noncompliant data ${ }^{\mathrm{d}}$ & $27(5) ; 560$ & $36(9) ; 394$ & $12(5) ; 260$ & $4(3) ; 123$ \\
\hline Refused to wear ${ }^{\mathrm{e}}$ & $3(<1) ; 571$ & $16(4) ; 414$ & $21(7) ; 284$ & $24(16) ; 154$ \\
\hline Unable to wear ${ }^{\mathrm{f}}$ & $8(1) ; 571$ & $4(1) ; 414$ & $3(1) ; 284$ & $5(3) ; 154$ \\
\hline Total inactive ${ }^{\mathrm{g}}$ & $9(2) ; 580$ & $166(28.6) ; 580$ & $296(51.0) ; 580$ & $384(71.6) ; 536$ \\
\hline Protocol noncompliant ${ }^{\mathrm{h}}$ & $\mathrm{N} / \mathrm{A}^{\mathrm{i}}$ & $14(8) ; 166$ & $25(8) ; 296$ & $27(7) ; 384$ \\
\hline Study endpoint ${ }^{\mathrm{j}}$ & $9(100) ; 9$ & 152 (91.6); 166 & 271 (91.6); 296 & 357 (93.0); 384 \\
\hline
\end{tabular}

\footnotetext{
${ }^{\mathrm{a}}$ Values may not add up to $100 \%$ because of rounding.

${ }^{\mathrm{b}}$ Active participants included those still on study time point and eligible to wear an activity monitor at each time point. At 24 months, 44 women were not yet at the study time point and, therefore, were not included in the sample size.

${ }^{\mathrm{c}}$ Agree to wear is defined as a participant who provided verbal consent and confirmation of availability to wear the accelerometer via phone.

${ }^{\mathrm{d}}$ Compliant data were defined as $\geq 4$ consecutive days, with $\geq 10$ hours of daily wear time. Percent is calculated by the number of all participants who provided verbal consent and were available to wear the accelerometer. Participants who were not active in the study were not included in the denominator for data compliance.

${ }^{\mathrm{e}}$ Refusal to wear is defined as a participant who declined to wear the accelerometer for any reason.

${ }^{\mathrm{f}}$ Unable to wear is defined as a participant who expressed willingness to wear the accelerometer but did not have the physical capacity to wear during the open window.

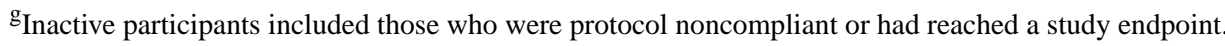

${ }^{\mathrm{h}}$ Protocol noncompliant is defined as a participant who did not return the accelerometer after 2 consecutive months of contact attempts.

${ }^{\mathrm{i}} \mathrm{N} / \mathrm{A}$ : not applicable.

${ }^{\mathrm{j}}$ Participants who reached a study endpoint, defined as disease progression, other diseases, lost to follow-up, or withdraw of participation, were not asked to wear the accelerometer at any future time points.
} 
Table 2. Baseline characteristics for women who were enrolled in the Lifestyle Intervention for oVarian cancer Enhanced Survival accelerometer protocol at baseline $(\mathrm{n}=580)$.

\begin{tabular}{|c|c|}
\hline Characteristics & Values \\
\hline Age at enrollment (years), mean (SD) & $60.07(9.3)$ \\
\hline \multicolumn{2}{|l|}{ Education $^{\mathrm{a}}, \mathrm{n}(\%)$} \\
\hline High school or less & $75(14.0)$ \\
\hline Some college education & $147(27.4)$ \\
\hline College graduate & $314(58.6)$ \\
\hline \multicolumn{2}{|l|}{ Ethnicity $^{\mathrm{a}}, \mathbf{n}(\%)$} \\
\hline Hispanic & $34(6.4)$ \\
\hline Non-Hispanic & $500(93.6)$ \\
\hline \multicolumn{2}{|l|}{ BMI class $\left(\mathrm{kg} / \mathrm{m}^{2}\right), \mathbf{n}(\%)$} \\
\hline Normal (18.5-24.9) & $211(36.4)$ \\
\hline Overweight (25.0-29.9) & $203(35.0)$ \\
\hline Obese $(\geq 30.0)$ & $166(28.6)$ \\
\hline
\end{tabular}

${ }^{\mathrm{a}}$ Missing data $<8 \%$.

Table 3. Predictors of baseline compliant data and association with demographic and anthropometric characteristics $(\mathrm{n}=522)$.

\begin{tabular}{|c|c|c|}
\hline Characteristics $^{\mathrm{a}, \mathrm{b}}$ & Odds ratio $(95 \% \mathrm{CI})$ & $P$ value \\
\hline Age & $1.00(0.95-1.05)$ & $>.99$ \\
\hline \multicolumn{3}{|l|}{ Education } \\
\hline High school or less & $1.0^{\mathrm{c}}$ & $\mathrm{N} / \mathrm{A}^{\mathrm{d}}$ \\
\hline Some college education & $3.27(0.74-14.44)$ & .12 \\
\hline College graduate & $2.60(0.68-9.92)$ & .16 \\
\hline \multicolumn{3}{|l|}{ Ethnicity } \\
\hline Non-Hispanic & 1.0 & N/A \\
\hline Hispanic & $0.89(0.15-5.25)$ & .90 \\
\hline \multicolumn{3}{|l|}{ BMI class } \\
\hline Normal & 1.0 & N/A \\
\hline Overweight & $1.17(0.33-4.13)$ & .80 \\
\hline Obese & $0.88(0.25-3.07)$ & .84 \\
\hline Tracking log completion & $54.71(17.05-175.59)$ & $<.001$ \\
\hline Mobile app engagement & $2.79(0.56-13.84)$ & .21 \\
\hline
\end{tabular}

${ }^{\mathrm{a}}$ Using data available for participants who agreed to wear an accelerometer at baseline.

${ }^{\mathrm{b}}$ Only participants with nonmissing values for all variables are included in the logistic regression model.

${ }^{\mathrm{c}}$ These are all provided with the exception of the referent group, which would not have a CI- this is the group we are comparing against to determine the OR and CI.

${ }^{\mathrm{d}} \mathrm{N} / \mathrm{A}$ : not applicable.

\section{Compliance With Tracking Logs and Mobile App Engagement (Self-Monitoring)}

At baseline, 88.6\% (496/560) of the tracking logs were returned. At subsequent time points, $83.0 \%$ (327/394) were returned at 6 months, $84.2 \%(219 / 260)$ were returned at 12 months, and $86.2 \%(106 / 123)$ were returned at 24 months. The most common reason reported for the removal of the Actigraph GT9X-Link on the tracking logs was shower or bath, aligned with the wear protocol. All other reasons for removal accounted for $<10 \%$ of the reported removal for all days and included allowing the monitor to charge, swimming, or medical examination. Completion of the tracking log was significantly associated with compliant accelerometer wear data at all time points $(P<.001$, Table 4). 
Table 4. Prevalence of tracking log completion and manufacturer mobile app engagement with compliant accelerometry data collection among the active Lifestyle Intervention for oVarian cancer Enhanced Survival participants who agreed to wear an accelerometer at each study time point.

\begin{tabular}{|c|c|c|}
\hline Time point ${ }^{\mathrm{a}, \mathrm{b}}$ & Value, $\mathrm{n}(\%)$ & $P$ value \\
\hline \multicolumn{3}{|l|}{ Baseline $(n=560)$} \\
\hline Tracking log & $496(88.6)$ & $<.001$ \\
\hline Mobile app & $198(35.4)$ & .002 \\
\hline Both & $187(33.4)$ & $<.001$ \\
\hline \multicolumn{3}{|l|}{6 months $(n=394)$} \\
\hline Tracking log & $327(83.0)$ & $<.001$ \\
\hline Mobile app & $78(19.8)$ & .06 \\
\hline Both & $60(15.2)$ & .03 \\
\hline \multicolumn{3}{|l|}{12 months, $n=260$} \\
\hline Tracking log & $219(84.2)$ & $<.001$ \\
\hline Mobile app & $41(15.8)$ & .53 \\
\hline Both & $35(13.5)$ & .16 \\
\hline \multicolumn{3}{|c|}{24 months $(n=123)$} \\
\hline Tracking log & $106(86.2)$ & $<.001$ \\
\hline Mobile app & $13(10.6)$ & .49 \\
\hline Both & $12(9.8)$ & .50 \\
\hline
\end{tabular}

${ }^{a}$ Percent values are calculated by dividing by the number of active participants who agreed to wear an accelerometer at each time point.

${ }^{\mathrm{b}}$ Analyses were conducted using the Pearson chi-square tests.

At baseline, 35.4\% (198/560) of the participants opted to use the mobile app; the average frequency of uploads during the 7-day wear period was 5 (median 6). At 6, 12, and 24 months, $19 \%(78 / 123), 15 \%(41 / 123)$, and $11 \%(13 / 123)$ of the participants repeated the use of the app, respectively. The average frequency of uploads at 6,12 , and 24 months was 5 (median 6), 6 (median 6), and 6 (median 6), respectively. The use of the mobile app was significantly associated with compliant data at baseline only $(P=.002)$. Completion of both the tracking $\log$ and mobile app upload was significantly associated with compliant accelerometry data collected at baseline and 6 months $(P=.001$ and $P=.03$, respectively). Fewer participants completed both the tracking log and uploaded data through the mobile app at the following 12- and 24-month time points, with the majority of participants choosing to complete the tracking log only.

\section{Discussion}

\section{Principal Findings}

Given the current interest in objective measurement of physical activity levels in cancer survivors and reports of over- and under-reporting of physical activity using validated self-reported measures, a rigorous protocol, which includes SOPs, to successfully send, receive, collect, and download accelerometer data is recommended. A 2018 review of accelerometer-based activity monitoring in cancer survivors suggested that current efforts lack standardization in relation to the methodology used and further details of accelerometer data collection methods are lacking, making replication at best challenging [15]. To address this gap in current evidence, this study provides an adaptable, detailed protocol for consideration across trials, particularly those that are distance-delivered. On the basis of our findings, these protocols should include frequent communication via telephone or email, multiple methods of instruction for how to use the accelerometer (eg, video, paper, and telephone-based education), and a detailed mail tracking system. To increase the likelihood of receiving compliant and usable data, protocols should include having the participants upload their data in real time or record wear time on a standardized tracking form. Here, we report the study protocol, implementation, and initial compliant data for using accelerometers in a large, multisite national lifestyle intervention in a trial of ovarian cancer survivors. Data were collected from participants in 48 US states for objective measurement of activity. The LIvES research team has developed a reproducible protocol that can be used by others for the implementation of accelerometers in future research trials.

\section{Comparison With Prior Works}

The majority (98\%) of the data were collected within the predesignated windows for data collection. Agreement to wear the accelerometer did decrease over time among all protocol active participants. Our goal was to collect data from all active women within the subsample at all time points. However, a small percentage of the women were protocol noncompliant at previous time points or refused or were unable to wear the accelerometer (Table 1). Of those who provided verbal consent and availability to wear the accelerometer, $95.2 \%$ (533/560), 90.9\% (358/394), 95.4\% (248/260), and $97.6 \%$ provided compliant data at baseline, 6,12 , and 24 months, respectively. 
Our compliance rates for accelerometry were similar to large-scale prospective cohort studies in older women with single time point measurements [36] and were above the $71.6 \%$ wear time compliance estimates from adults wearing thigh and back-placed accelerometers [39] and much higher than estimates of $62.6 \%$ among children who participated in the National Health and Nutrition Examination Survey [40]. Compliant data on distance-delivered methodologies are sparse.

At the time of the development of this protocol, the GT9X was validated only for hip-worn measurements. Although the GT9X has since been validated for wristwear [18], it is unknown how this may have affected compliance in our sample. Among a large sample of European adults with a single time point of collection, $93.3 \%$ had valid data for wrist-worn accelerometers [41]. Comparatively, we demonstrated $95.2 \%$ valid compliant data from hip-worn accelerometers in our sample at baseline. The current literature suggests a poor correlation between wrist and hip-worn accelerometer counts per min; therefore, comparison of estimates from wear at the 2 different sites should be interpreted with caution $[42,43]$. To maintain consistency in the data collected, all participants were asked to wear GT9X on their nondominant hip at all time points. Furthermore, the hip placement of GT9X demonstrates better step count accuracy [18], which is an a priori behavior outcome for the trial. In addition, the quality of the data generated using the protocol will allow for time in sedentary bouts, light activity, and the development of population-specific cut-off points that accommodate the described reduction in peak oxygen consumption and relative intensity of activities among cancer survivors $[44,45]$.

Contrary to other studies that may suggest that obese women have higher nonwear time of accelerometers [46], our results indicate that BMI did not have a significant effect on compliant data in ovarian cancer survivors. Tracking log completion remained strongly associated with compliant wear data across all time points. Calls at 6,12 , and 24 months became more streamlined and shorter in length, as many participants were already familiar with the protocol and felt comfortable with the device without additional telephone support at these time points. High compliance overall may reflect the older female sample of cancer survivors motivated to participate in a 24-month lifestyle intervention.

The use of the mobile app to upload data was optional, and $35.4 \%$ of women opted to use this app. We noted that age, though not statistically significant, influenced the use of the mobile app, suggesting that this approach may have higher adherence for younger cancer survivors. The mobile app allowed participants to upload data but they could not see their activity data to keep data collection blinded. This may have influenced the motivation for repeated app engagement. Over time, participants engaged less with the mobile app, results that are similar to the patterns previously reported in the literature for other health-related smartphone apps [47]. This warrants further studies, including new strategies to promote the continued use of technology. Of note, our instruction call notes from participants indicated that many participants did not find any benefit or did not feel like there was time available to complete both the tracking $\log$ and mobile app. An additional consideration in applying the findings of this research is our focus on a convenience sample of ovarian cancer survivors. Although we anticipate that the protocol will perform similarly in other cancer survivor populations, this is not a standard, particularly in relation to diversity in sex and education as well as cancer-related symptom burden and comorbidities.

\section{Future Directions}

Beyond the scope of this protocol, but important to future research in this area, population-relevant cut-off points and algorithms need to be established for cancer survivors for both wrist and hip-worn accelerometers. The field of accelerometry is rapidly emerging, especially regarding standardized cut-off points. For data analysis related to objective physical activity data captured in this protocol, standard cut-off points as well as vector magnitude and total activity counts will be evaluated by the research team. The current literature suggests that vector magnitude may better discriminate between sedentary and light physical activity in women aged older than 60 years [48], although among breast cancer survivors, total activity counts may provide better estimates of moderate-to-vigorous physical activity [49]. These population-relevant cut-off points will allow for a more accurate interpretation of accelerometer data for the older female cancer survivor population; however, information remains limited for cut-off points specific to ovarian cancer survivors. Importantly, both age and the presence of comorbidities can influence accelerometer cut-off points [48], confounders that are highly relevant to this population.

In summary, we have developed a detailed protocol and related materials for collecting the accelerometry data from a large sample of cancer survivors who reside across the United States. This protocol has resulted in the acquisition of a robust data set for future analysis of physical activity in this population. This protocol and the related materials that were issued to participants are available through UACC-BMISR consultation services [50] in support of future research studies designed to capture repeated measures of activity in this vulnerable population.

\section{Acknowledgments}

The authors wish to thank all the work and efforts of the staff and the undergraduate and graduate student research team who worked on this project from its inception: Natasha Burrell, Kelly Carroll, Sarah Lavelle, Sophia Perkin, Jennifer Shipley, McKenna Scheldt, Madeline Schneider, Patrice Tillman, Amanda Younger, and Samantha Werts. We also thank the 580 ovarian cancer survivors who dedicated their time and provided data for this research. This work was supported by the National Cancer Institute grants to NRG Oncology (U10 CA 180822), NRG operations (U10 CA180868), and UG1CA189867 (NCORP). Additional funding for this research was provided by the NRG Grant (R305B160003), the National Institutes of Health National Cancer Institute (1R01 CA186700), the BMISR at the UACC Support Grant (P30 CA023074), and the West Valley Ovarian Cancer Alliance. 


\section{Conflicts of Interest}

None declared.

\section{References}

1. Mishra SI, Scherer RW, Geigle PM, Berlanstein DR, Topaloglu O, Gotay CC, et al. Exercise interventions on health-related quality of life for cancer survivors. Cochrane Database Syst Rev 2012 Aug 15(8):CD007566 [FREE Full text] [doi: 10.1002/14651858.CD007566.pub2] [Medline: 22895961]

2. Garcia DO, Thomson CA. Physical activity and cancer survivorship. Nutr Clin Pract 2014 Dec;29(6):768-779 [FREE Full text] [doi: 10.1177/0884533614551969] [Medline: 25335787]

3. Smits A, Smits E, Lopes A, Das N, Hughes G, Talaat A, et al. Body mass index, physical activity and quality of life of ovarian cancer survivors: time to get moving? Gynecol Oncol 2015 Oct;139(1):148-154. [doi: 10.1016/j.ygyno.2015.08.005] [Medline: 26291650]

4. Mizrahi D, Naumann F, Broderick C, Samara J, Ryan M, Friedlander M. Quantifying physical activity and the associated barriers for women with ovarian cancer. Int J Gynecol Cancer 2015 May;25(4):577-583. [doi:

10.1097/IGC.0000000000000349] [Medline: 25628105]

5. Kushi LH, Doyle C, McCullough M, Rock CL, Demark-Wahnefried W, Bandera EV, American Cancer Society 2010 Nutrition and Physical Activity Guidelines Advisory Committee. American cancer society guidelines on nutrition and physical activity for cancer prevention: reducing the risk of cancer with healthy food choices and physical activity. CA Cancer J Clin 2012;62(1):30-67 [FREE Full text] [doi: 10.3322/caac.20140] [Medline: 22237782]

6. Irwin ML, Ainsworth BE. Physical activity interventions following cancer diagnosis: methodologic challenges to delivery and assessment. Cancer Invest 2004;22(1):30-50. [doi: 10.1081/cnv-120027579] [Medline: 15069762]

7. Vassbakk-Brovold K, Kersten C, Fegran L, Mjåland O, Mjåland S, Seiler S, et al. Cancer patients participating in a lifestyle intervention during chemotherapy greatly over-report their physical activity level: a validation study. BMC Sports Sci Med Rehabil 2016;8:10 [FREE Full text] [doi: 10.1186/s13102-016-0035-z] [Medline: 27099757]

8. Dyrstad SM, Hansen BH, Holme IM, Anderssen SA. Comparison of self-reported versus accelerometer-measured physical activity. Med Sci Sports Exerc 2014 Jan;46(1):99-106. [doi: 10.1249/MSS.0b013e3182a0595f] [Medline: 23793232]

9. Dowd KP, Szeklicki R, Minetto MA, Murphy MH, Polito A, Ghigo E, et al. A systematic literature review of reviews on techniques for physical activity measurement in adults: a DEDIPAC study. Int J Behav Nutr Phys Act 2018 Feb 8;15(1):15 [FREE Full text] [doi: 10.1186/s12966-017-0636-2] [Medline: 29422051]

10. Thraen-Borowski KM, Gennuso KP, Cadmus-Bertram L. Accelerometer-derived physical activity and sedentary time by cancer type in the United States. PLoS One 2017;12(8):e0182554 [FREE Full text] [doi: 10.1371/journal.pone.0182554] [Medline: 28806753]

11. Shiroma EJ, Cook NR, Manson JE, Buring JE, Rimm EB, Lee I. Comparison of self-reported and accelerometer-assessed physical activity in older women. PLoS One 2015;10(12):e0145950 [FREE Full text] [doi: 10.1371/journal.pone.0145950] [Medline: 26713857]

12. Zhong S, Chen L, Lv M, Ma T, Zhang X, Zhao J. Nonoccupational physical activity and risk of ovarian cancer: a meta-analysis. Tumour Biol 2014 Nov;35(11):11065-11073. [doi: 10.1007/s13277-014-2385-z] [Medline: 25095977]

13. Hildebrand JS, Gapstur SM, Gaudet MM, Campbell PT, Patel AV. Moderate-to-vigorous physical activity and leisure-time sitting in relation to ovarian cancer risk in a large prospective US cohort. Cancer Causes Control 2015 Nov;26(11):1691-1697. [doi: 10.1007/s10552-015-0656-7] [Medline: 26335264]

14. Montoye AH, Moore RW, Bowles HR, Korycinski R, Pfeiffer KA. Reporting accelerometer methods in physical activity intervention studies: a systematic review and recommendations for authors. Br J Sports Med 2018 Dec;52(23):1507-1516. [doi: 10.1136/bjsports-2015-095947] [Medline: 27539504]

15. Peddle-McIntyre CJ, Cavalheri V, Boyle T, McVeigh JA, Jeffery E, Lynch BM, et al. A review of accelerometer-based activity monitoring in cancer survivorship research. Med Sci Sports Exerc 2018 Sep;50(9):1790-1801. [doi: 10.1249/MSS.0000000000001644] [Medline: 29683922]

16. Stavraka C, Ford A, Ghaem-Maghami S, Crook T, Agarwal R, Gabra H, et al. A study of symptoms described by ovarian cancer survivors. Gynecol Oncol 2012 Apr;125(1):59-64. [doi: 10.1016/j.ygyno.2011.12.421] [Medline: 22155797]

17. Zhou Y, Irwin M, Ferrucci L, McCorkle R, Ercolano E, Li F, et al. Health-related quality of life in ovarian cancer survivors: results from the American cancer society's study of cancer survivors - I. Gynecol Oncol 2016 Jun;141(3):543-549 [FREE Full text] [doi: 10.1016/j.ygyno.2016.04.006] [Medline: 27072805]

18. Hwang J, Fernandez AM, Lu AS. Application and validation of activity monitors' epoch lengths and placement sites for physical activity assessment in exergaming. J Clin Med 2018 Sep 11;7(9):- [FREE Full text] [doi: 10.3390/jcm7090268] [Medline: 30208567]

19. ActiGraph Link GT9X. ActiGraph. 2018. URL: https://www.actigraph.nl/en/product/72/actigraph-link-gt9x.html [accessed 2019-08-03] 
20. van Remoortel H, Raste Y, Louvaris Z, Giavedoni S, Burtin C, Langer D, PROactive Consortium. Validity of six activity monitors in chronic obstructive pulmonary disease: a comparison with indirect calorimetry. PLoS One 2012;7(6):e39198 [FREE Full text] [doi: 10.1371/journal.pone.0039198] [Medline: 22745715]

21. John D, Morton A, Arguello D, Lyden K, Bassett D. 'What is a step?' differences in how a step is detected among three popular activity monitors that have impacted physical activity research. Sensors (Basel) 2018 Apr 15;18(4):- [FREE Full text] [doi: 10.3390/s18041206] [Medline: 29662048]

22. Montoye AH, Nelson MB, Bock JM, Imboden MT, Kaminsky LA, Mackintosh KA, et al. Raw and count data comparability of hip-worn actigraph GT3X+ and link accelerometers. Med Sci Sports Exerc 2018 May;50(5):1103-1112. [doi: 10.1249/MSS.0000000000001534] [Medline: 29283934]

23. Jaarsma T, Klompstra L, Ben Gal T, Boyne J, Vellone E, Bäck M, et al. Increasing exercise capacity and quality of life of patients with heart failure through Wii gaming: the rationale, design and methodology of the HF-Wii study; a multicentre randomized controlled trial. Eur J Heart Fail 2015 Jul;17(7):743-748 [FREE Full text] [doi: 10.1002/ejhf.305] [Medline: 26139585]

24. Noonan RJ, Fairclough SJ, Knowles ZR, Boddy LM. Context matters! sources of variability in weekend physical activity among families: a repeated measures study. BMC Public Health 2017 Apr 18;17(1):330 [FREE Full text] [doi: 10.1186/s12889-017-4232-9] [Medline: 28420363]

25. Coker-Bolt P, Downey RJ, Connolly J, Hoover R, Shelton D, Seo NJ. Exploring the feasibility and use of accelerometers before, during, and after a camp-based CIMT program for children with cerebral palsy. J Pediatr Rehabil Med 2017;10(1):27-36. [doi: 10.3233/PRM-170408] [Medline: 28339408]

26. Thomson CA, Crane TE, Miller A, Garcia DO, Basen-Engquist K, Alberts DS. A randomized trial of diet and physical activity in women treated for stage II-IV ovarian cancer: rationale and design of the lifestyle intervention for ovarian cancer enhanced survival (LIVES): an NRG oncology/gynecologic oncology group (GOG-225) study. Contemp Clin Trials 2016 Jul;49:181-189 [FREE Full text] [doi: 10.1016/j.cct.2016.07.005] [Medline: 27394382]

27. Staten LK, Taren DL, Howell WH, Tobar M, Poehlman ET, Hill A, et al. Validation of the Arizona activity frequency questionnaire using doubly labeled water. Med Sci Sports Exerc 2001 Nov;33(11):1959-1967. [doi: 10.1097/00005768-200111000-00024] [Medline: $\underline{11689750]}$

28. Lee I, Shiroma EJ. Using accelerometers to measure physical activity in large-scale epidemiological studies: issues and challenges. Br J Sports Med 2014 Mar;48(3):197-201 [FREE Full text] [doi: 10.1136/bjsports-2013-093154] [Medline: 24297837]

29. Troiano RP, McClain JJ, Brychta RJ, Chen KY. Evolution of accelerometer methods for physical activity research. Br J Sports Med 2014 Jul;48(13):1019-1023 [FREE Full text] [doi: 10.1136/bjsports-2014-093546] [Medline: 24782483]

30. Herrmann SD, Barreira TV, Kang M, Ainsworth BE. Impact of accelerometer wear time on physical activity data: a NHANES semisimulation data approach. Br J Sports Med 2014 Mar;48(3):278-282. [doi: 10.1136/bjsports-2012-091410] [Medline: 22936409]

31. Broderick JM, Ryan J, O'Donnell DM, Hussey J. A guide to assessing physical activity using accelerometry in cancer patients. Support Care Cancer 2014 Apr;22(4):1121-1130. [doi: 10.1007/s00520-013-2102-2] [Medline: 24389829]

32. LaCroix AZ, Rillamas-Sun E, Buchner D, Evenson KR, Di C, Lee I, et al. The objective physical activity and cardiovascular disease health in older women (OPACH) study. BMC Public Health 2017 Feb 14;17(1):192 [FREE Full text] [doi: 10.1186/s12889-017-4065-6] [Medline: 28193194]

33. Crane TE, Parizek D, Eddy N, Franks GH, Yung AK, Merchant N, inventors, University of Arizona, assignee. Ehealth and intervention platform. United States patent application US 15/655,553. 2018 Jan 25.

34. CentrePoint Mobile User Manual. ActiGraph. 2018. URL: https://actigraph.desk.com/customer/en/portal/articles/ 2515621-centrepoint-mobile-user-manual [accessed 2019-08-03]

35. Lyden K, Kozey SL, Staudenmeyer JW, Freedson PS. A comprehensive evaluation of commonly used accelerometer energy expenditure and MET prediction equations. Eur J Appl Physiol 2011 Mar;111(2):187-201 [FREE Full text] [doi: 10.1007/s00421-010-1639-8] [Medline: 20842375]

36. LaMonte MJ, Buchner DM, Rillamas-Sun E, Di C, Evenson KR, Bellettiere J, et al. Accelerometer-measured physical activity and mortality in women aged 63 to 99. J Am Geriatr Soc 2018 May;66(5):886-894 [FREE Full text] [doi: 10.1111/jgs.15201] [Medline: 29143320]

37. Sweegers MG, Boyle T, Vallance JK, Chinapaw MJ, Brug J, Aaronson NK, et al. Which cancer survivors are at risk for a physically inactive and sedentary lifestyle? Results from pooled accelerometer data of 1447 cancer survivors. Int J Behav Nutr Phys Act 2019 Aug 16;16(1):66 [FREE Full text] [doi: 10.1186/s12966-019-0820-7] [Medline: 31420000]

38. Freedson PS, Melanson E, Sirard J. Calibration of the computer science and applications inc accelerometer. Med Sci Sports Exerc 1998 May;30(5):777-781. [doi: 10.1097/00005768-199805000-00021] [Medline: 9588623]

39. Duncan S, Stewart T, Mackay L, Neville J, Narayanan A, Walker C, et al. Wear-time compliance with a dual-accelerometer system for capturing 24-h behavioural profiles in children and adults. Int J Environ Res Public Health 2018 Jun 21;15(7):[FREE Full text] [doi: 10.3390/ijerph15071296] [Medline: 29933548]

40. Tudor-Locke C, Barreira TV, Schuna JM, Mire EF, Chaput J, Fogelholm M, ISCOLE Research Group. Improving wear time compliance with a 24-hour waist-worn accelerometer protocol in the international study of childhood obesity, lifestyle 
and the environment (ISCOLE). Int J Behav Nutr Phys Act 2015 Mar 11;12:11 [FREE Full text] [doi:

10.1186/s12966-015-0172-x] [Medline: 25881074]

41. Doherty A, Jackson D, Hammerla N, Plötz T, Olivier P, Granat MH, et al. Large scale population assessment of physical activity using wrist worn accelerometers: the UK biobank study. PLoS One 2017;12(2):e0169649 [FREE Full text] [doi: 10.1371/journal.pone.0169649] [Medline: 28146576]

42. Tudor-Locke C, Barreira TV, Schuna JM. Comparison of step outputs for waist and wrist accelerometer attachment sites. Med Sci Sports Exerc 2015 Apr;47(4):839-842. [doi: 10.1249/MSS.0000000000000476] [Medline: 25121517]

43. Loprinzi PD, Smith B. Comparison between wrist-worn and waist-worn accelerometry. J Phys Act Health 2017 Jul;14(7):539-545. [doi: 10.1123/jpah.2016-0211] [Medline: 28290761]

44. Kampshoff CS, Chinapaw MJ, Brug J, Twisk JW, Schep G, Nijziel MR, et al. Randomized controlled trial of the effects of high intensity and low-to-moderate intensity exercise on physical fitness and fatigue in cancer survivors: results of the resistance and endurance exercise after chemotherapy (REACT) study. BMC Med 2015 Oct 29;13:275 [FREE Full text] [doi: 10.1186/s12916-015-0513-2] [Medline: 26515383]

45. Miller NE, Strath SJ, Swartz AM, Cashin SE. Estimating absolute and relative physical activity intensity across age via accelerometry in adults. J Aging Phys Act 2010 Apr;18(2):158-170 [FREE Full text] [doi: 10.1123/japa.18.2.158] [Medline: 20440028]

46. Tudor-Locke C, Brashear MM, Johnson WD, Katzmarzyk PT. Accelerometer profiles of physical activity and inactivity in normal weight, overweight, and obese US men and women. Int J Behav Nutr Phys Act 2010 Aug 3;7:60 [FREE Full text] [doi: 10.1186/1479-5868-7-60] [Medline: 20682057]

47. Krebs P, Duncan DT. Health app use among US mobile phone owners: a national survey. JMIR Mhealth Uhealth 2015;3(4):e101 [FREE Full text] [doi: 10.2196/mhealth.4924] [Medline: 26537656]

48. Evenson KR, Wen F, Herring AH, Di C, LaMonte MJ, Tinker LF, et al. Calibrating physical activity intensity for hip-worn accelerometry in women age 60 to 91 years: the women's health initiative OPACH calibration study. Prev Med Rep 2015;2:750-756 [FREE Full text] [doi: 10.1016/j.pmedr.2015.08.021] [Medline: 26527313]

49. Trinh L, Motl RW, Roberts SA, Gibbons T, McAuley E. Estimation of physical activity intensity cut-points using accelerometry in breast cancer survivors and age-matched controls. Eur J Cancer Care (Engl) 2019 Sep;28(5):e13090. [doi: 10.1111/ecc.13090] [Medline: $\underline{31106924]}$

50. Behavioral Measurement and Interventions Shared Resource. University of Arizona Cancer Center. 2018. URL: https:/ /cancercenter.arizona.edu/researchers/shared-resources/behavioral-measurement-and-interventions [accessed 2020-04-19]

\author{
Abbreviations \\ APAQ: Arizona Physical Activity Questionnaire \\ BMISR: Behavioral Measurement and Interventions Shared Resource \\ HIPAA: Health Insurance Portability and Accountability Act \\ LIvES: Lifestyle Intervention for oVarian cancer Enhanced Survival \\ OR: odds ratio \\ SOP: standard operating procedure \\ UACC: University of Arizona Cancer Center \\ USPS: United States Postal Service
}

Edited by G Eysenbach; submitted 28.02.20; peer-reviewed by J Brown, C Valle; comments to author 08.04.20; revised version
received 03.06.20; accepted 26.07.20; published 24.09.20
Please cite as:
Crane TE, Skiba MB, Miller A, Garcia DO, Thomson CA
Development and Evaluation of an Accelerometer-Based Protocol for Measuring Physical Activity Levels in Cancer Survivors:
Development and Usability Study
JMIR Mhealth Uhealth 2020;8(9):e18491
URL: $\underline{\text { http://mhealth.jmir.org/2020/9/e18491/ }}$
doi: $\underline{10.2196 / 18491}$
PMID: $\underline{32969828}$

(C)Tracy E Crane, Meghan B Skiba, Austin Miller, David O Garcia, Cynthia A Thomson. Originally published in JMIR mHealth and uHealth (http://mhealth.jmir.org), 24.09.2020. This is an open-access article distributed under the terms of the Creative Commons Attribution License (https://creativecommons.org/licenses/by/4.0/), which permits unrestricted use, distribution, and reproduction in any medium, provided the original work, first published in JMIR mHealth and uHealth, is properly cited. The 
complete bibliographic information, a link to the original publication on http://mhealth.jmir.org/, as well as this copyright and license information must be included. 